Note CRAS project

Équations aux dérivées partielles/Partial Differential Equations

(Systèmes dynamiques/Dynamical Systems)

\title{
Global attractors for multivalued random semiflows gener- ated by random differential inclusions with additive noise
}

\author{
Tomás CARABALLO ${ }^{\text {a }}$, José A. LANGA ${ }^{a}$, José VALERO ${ }^{b}$ \\ a Dpto. Ecuaciones Diferenciales y Análisis Numérico, Universidad de Sevilla, Apdo. Correos 1160, \\ 41080-Sevilla (Spain) E-mails : caraball@numer.us.es ; langa@numer.us.es \\ b Universidad Cardenal Herrera CEU, Comisario 3, 03203-Elche, Alicante (Spain)
}

\begin{abstract}
We introduce the concept of multivalued random dynamical system (MRDS) as a measurable multivalued flow satisfying the cocycle property. We show how this is a suitable framework for the study of the asymptotic behaviour of some multivalued stochastic parabolic equations by generalizing the concept of global random attractor to the case of a MRDS. (c) Académie des Sciences/Elsevier, Paris
Attracteurs globaux pour des semi-flots aléatoires multivalués engendrés par des inclusions différentielles stochastiques avec un bruit additif

Résumé. Dans cette Note, on présente la notion de Système Dynamique Aléatoire Multivalué (MRDS) comme un flot mesurable multivalué qui satisfait la propriété du cocycle. Par une généralisation du concept d'attracteur global aléatoire dans le cas d'un MRDS, on montre que cette notion est bien adaptée pour l'étude du comportement asymptotique de quelques equations stochastiques paraboliques multivaluées. (c) Académie des Sciences/Elsevier, Paris
\end{abstract}

\section{French Abridged Version}

Cette Note a comme objectif fondamental l'analyse du comportement asymptotique de quelques inclusions différentielles stochastiques. D'abord, nous développons une théorie similaire à celle de Crauel et Flandoli [8] pour le cas des systèmes dynamiques univalués. Nous introduisons la notion de Système Dynamique Aléatoire Multivalué (MRDS), ou Semi-flot Aléatoire Multivalué, comme une multi-application mesurable qui satisfait la propriété du cocycle (Definition 1). Ensuite, nous démontrons que l'existence d'un ensemble aléatoire absorbant et compact, ainsi que la semicontinuité supérieure du MRDS, impliquent l'existence d'un d'attracteur global aléatoire (Théorème 2 et Definition 4).

Au paragraphe 3, nous étudions une inclusion différentielle stochastique avec un bruit additif (voir (1)), et nous démontrons que, sous certaines hypothèses sur les opérateurs $A$ et $F$ (surtout, m-dissipativité pour $A$ et caractère lipschitzien pour $F$ ), l'inclusion différentielle (1) engendre un MRDS. La construction est basée sur un changement de variables adéquat, qui tranforme l'inclusion stochastique dans une autre inclusion déterministe dépendant d'un paramètre, à laquelle on peut appliquer les résultats de la théorie déterministe. Utilisant la notion de solution intégrale du 
système transformé (voir Definitions 5-7), nous montrons comment on peut construire un semi-flot aléatoire multivalué.

Pour terminer, sous une hypothèse de dissipativité, et une condition de compacité sur le MRDS, nous démontrons l'existence d'un ensemble aléatoire absorbant et compact, ce qui assure l'existence d'un attracteur global aléatoire.

Une étude plus detaillée, avec des applications à des inclusions stochastiques de réaction-diffusion, sera publiée prochainement (voir [6]).

\section{Introduction}

The study of the qualitative behaviour of ordinary and partial differential equations is one of the most developed branches in this field. When a phenomenon from Physics, Chemistry, Biology, Economics can be described by a system of differential equations where the existence of global solutions can be assured, one of the most interesting problems is to know what is the asymptotic behaviour of the system when time grows to infinite. The study of the asymptotic behaviour of the system is giving us relevant information about "the future" of the phenomenon described in the model. In this context, the concept of global attractor has become a very useful tool to describe the long-time behaviour of many important differential equations (see, among others, Ladyzhenkaya [11], Hale [10], Temam [14]).

However, some difficulties appear when we have to work without uniqueness of solutions in the system or when the model is better described by, for instance, a differential inclusion. In these cases, it has been shown that the theory of multivalued flows makes suitable the treatment of the asymptotic behaviour of these differential equations and inclusions (Melnik and Valero [12], Valero $[16])$.

A new and different difficulty appears when a random term is added to the deterministic equation, a white noise for instance, so that the corresponding stochastic partial differential equation must be treated in a different way. Firstly, the equation becomes nonautonomous, which makes necessary the introduction of a process instead of a semigroup. Moreover, the strong dependence on the random term adds another difficulty. The new and rapidly growing theory of random dynamical systems (Arnold [2]) has become the appropriate tool for the study of many important random and stochastic equations. In this framework, Crauel and Flandoli [8] (see also Schmalfuss [13]) introduced the concept of random attractor as a proper generalization (see Caraballo et al. [5]) of the corresponding (deterministic) global attractor.

The joint treatment of multivalued functions and stochastic terms in a differential equation makes difficult even the existence and uniqueness of solutions for these systems (Ahmed [1], Da Prato and Frankowska [9], among others). In this paper we show how some stochastic differential inclusions generate multivalued random semiflows or multivalued random dynamical systems (MRDS) and study the asymptotic behaviour of some stochastic differential inclusions by previously introducing the corresponding concept of random attractor for this case. The proofs of the results in this Note and a detailed analysis of the problem (including some applications) can be found in [6].

\section{Multivalued random dynamical systems and attractors}

Let $\left(X, d_{X}\right)$ be a complete and separable metric space with the Borel $\sigma$-algebra $\mathcal{B}(X)$. Let $(\Omega, \mathcal{F}, \mathbf{P})$ be a probability space and $\theta_{t}: \Omega \rightarrow \Omega$ a measure preserving group of transformations in $\Omega$ such that the map $(t, \omega) \mapsto \theta_{t} \omega$ is measurable and satisfying

$$
\theta_{t+s}=\theta_{t} \circ \theta_{s}=\theta_{s} \circ \theta_{t} ; \quad \theta_{0}=I d .
$$


The parameter $t$ takes values in $\mathbf{R}$ endowed with the Borel $\sigma$-algebra $\mathcal{B}(\mathbf{R})$.

Definition 1. - A set valued map $G: \mathbf{R}^{+} \times \Omega \times X \rightarrow C(X)(C(X)$ denotes the set of non-empty closed subsets of $X$ ) is called a multivalued random dynamical system (MRDS) or a multivalued random semiflow if is measurable (see Aubin and Frankowska [4], definition 8.1.1) and it satisfies

i) $G(0, \omega)=I d$ on $X$;

ii) $G(t+s, \omega) x=G\left(t, \theta_{s} \omega\right) G(s, \omega) x$ (cocycle property) for all $t, s \in \mathbf{R}^{+}, x \in X, \omega \in \Omega$.

Remark. When ii) holds identically, we call $G$ a perfect cocycle. We call $G$ a crude cocycle if ii) holds for fixed $s$ and all $t \in \mathbf{R}^{+}, x \in X, \mathbf{P}-$ a.s. (where the exceptional set $N_{s}$ can depend on $s$ ). We call $G$ a very crude cocycle if ii) holds for fixed $s, t \in \mathbf{R}^{+}$, for all $x \in X, \mathbf{P}-$ a.s. (where the exceptional set $N_{s, t}$ can depend on both $s$ and $t$ ).

Definition 2. - The MRDS $G$ is said to be upper semicontinuous if for all $t \in \mathbf{R}^{+}$and $\omega \in \Omega$ it follows that given $x \in X$ and a neighbourhood of $G(t, \omega) x, \mathcal{O}(G(t, \omega) x)$, there exists $\delta>0$ such that if $d_{X}(x, y)<\delta$, then $G(t, \omega) y \subset \mathcal{O}(G(t, \omega) x)$.

On the other hand, $G$ is called lower semicontinuous if for all $t \in \mathbf{R}^{+}$and $\omega \in \Omega$, given $x_{n} \rightarrow x$ $(n \rightarrow+\infty)$ and $y \in G(t, \omega) x$, there exists $y_{n} \in G(t, \omega) x_{n}$ such that $y_{n} \rightarrow y$.

It is said to be continuous if it is upper and lower semicontinuous.

Now, we extend the concept of random attractor to the case of a MRDS and show a general result for the existence and uniqueness of attractors. Firstly we need some definitions.

Definition 3. - A closed random set $D$ is a measurable map $D: \Omega \rightarrow C(X)$ in the sense of Castaing and Valadier [7], that is, given $x \in X$ the map $\omega \in \Omega \mapsto \operatorname{dist}(x, D(\omega))$ is measurable.

A closed random set $D(\omega)$ is said to be negatively (resp. strictly) invariant for the MRDS $G$ if $D\left(\theta_{t} \omega\right) \subset G(t, \omega) D(\omega)\left(\right.$ resp. $\left.D\left(\theta_{t} \omega\right)=G(t, \omega) D(\omega)\right), \quad \forall t \in \mathbf{R}^{+}, \omega \in \Omega$.

Let us assume the following conditions for the MRDS $G$ :

(H1) There exists an absorbing random compact set $B(\omega)$, that is, for $\mathbf{P}$-almost all $\omega \in \Omega$ and every bounded set $D \subset X$, there exists $t_{D}(\omega)$ such that $G\left(t, \theta_{-t} \omega\right) D \subset B(\omega)$, for all $t \geq t_{D}(\omega)$.

(H2) $G(t, \omega): X \rightarrow C(X)$ is upper semicontinuous, for all $t \in \mathbf{R}^{+}$and $\omega \in \Omega$.

Define the limit set of a bounded set $D \subset X$ as

$$
\Lambda_{D}(\omega)=\cap_{T \geq 0} \overline{\cup_{t \geq T} G\left(t, \theta_{-t} \omega\right) D}
$$

We now have the following proposition concerning some properties of the set $\Lambda_{D}(\omega)$ :

Proposition 1. - Assume conditions (H1) and (H2) hold. Then, for $\mathbf{P}$-almost all $\omega \in \Omega$ and every $D \subset X$ bounded, it follows:

i) $\Lambda_{D}(\omega) \subset B(\omega)$ is nonvoid and compact.

ii) $\Lambda_{D}(\omega)$ is negatively invariant. If in addition $G$ is lower semicontinuous, then $\Lambda_{D}(\omega)$ is strictly invariant.

iii) $\Lambda_{D}(\omega)$ attracts $D$, i.e.,

$$
\lim _{t \rightarrow+\infty} \operatorname{dist}\left(G\left(t, \theta_{-t} \omega\right) D, \Lambda_{D}(\omega)\right)=0
$$

DEFINITION 4. - The closed random set $\omega \mapsto \mathcal{A}(\omega)$ is a global random attractor for the MRDS $G$ if $\mathbf{P}-$ a.s.

a) $G(t, \omega) \mathcal{A}(\omega)=\mathcal{A}\left(\theta_{t} \omega\right)$, for all $t \geq 0$, (that is, it is strictly invariant); 
T. Caraballo, J.A. Langa, J. Valero

b) for all bounded $D \subset X$,

$$
\lim _{t \rightarrow+\infty} \operatorname{dist}\left(G\left(t, \theta_{-t} \omega\right) D, \mathcal{A}(\omega)\right)=0
$$

c) $\mathcal{A}(\omega)$ is compact.

We can now state the following theorem on the existence of random attractors for MRDS:

Theorem 2. - Let (H1) - (H2) hold, the map $(t, \omega) \in \mathbf{R}^{+} \times \Omega \mapsto \overline{G(t, \omega) D}$ be measurable for all deterministic bounded sets $D \subset X$, and the map $x \in X \mapsto G(t, \omega) x$ have compact values. Then,

$$
\mathcal{A}(\omega)=\overline{\cup_{\text {bounded } D \subset X} \Lambda_{D}(\omega)}
$$

is a global random attractor for $G$ (measurable with respect to $\mathcal{F}$ ). It is unique and the minimal closed attracting set.

\section{MRDS generated by a stochastic differential inclusion with additive noise}

\subsection{Generation}

Let $X$ be a real separable Hilbert space with the scalar product $\langle\cdot, \cdot\rangle$ and the norm $\|\cdot\|$. Consider the following stochastic differential inclusion

$$
\left\{\begin{array}{l}
\frac{d u}{d t} \in A u(t)+F(u(t))+\sum_{i=1}^{m} \phi_{i} \frac{d w_{i}(t)}{d t}, \quad t \in(0, T), \\
u(0)=u_{0},
\end{array}\right.
$$

where $A: D(A) \rightarrow X$ is a linear operator, $\phi_{i} \in D(A)$ and $w_{i}(t)$ are independent two-sided, i.e. $t \in \mathbf{R}$, real Wiener processes with $w_{i}(0)=0, i=1, \ldots, m$.

Let us introduce the next conditions:

(A) The operator $A$ is m-dissipative, i.e. $\forall y \in D(A),\langle A y, y\rangle \leq 0$, and $\operatorname{Im}(A-\lambda I)=X, \forall \lambda>0$.

(F1) $F: X \rightarrow C_{v}(X)$, where $C_{v}(X)$ is the set of all non-empty, bounded, closed, convex subsets of $X$.

(F2) The map $F$ is Lipschitz on $\overline{D(A)}$, i.e. $\exists C \geq 0$ such that $\forall y_{1}, y_{2} \in \overline{D(A)}$

$$
\operatorname{dist}_{H}\left(F\left(y_{1}\right), F\left(y_{2}\right)\right) \leq C\left\|y_{1}-y_{2}\right\|,
$$

where $\operatorname{dist}_{H}(\cdot, \cdot)$ denotes the Hausdorff metric of bounded sets.

Let $\zeta(t)=\sum_{i=1}^{m} \phi_{i} w_{i}(t)$. Let us consider the Wiener probability space $(\Omega, \mathcal{F}, \mathbf{P})$ defined by $\Omega=\left\{\omega=\left(w_{1}(\cdot), \ldots, w_{m}(\cdot)\right) \in C\left(\mathbf{R}, \mathbf{R}^{m}\right) \mid \omega(0)=0\right\}$, equipped with the Borel $\sigma$-algebra $\mathcal{F}$, the Wiener measure $\mathbf{P}$, and the usual uniform convergence on bounded sets of $\mathbf{R}$. Each $\omega \in \Omega$ generates a map $\zeta(\cdot)=\sum_{i=1}^{m} \phi_{i} w_{i}(\cdot) \in C(\mathbf{R}, X)$ such that $\zeta(0)=0$.

Making the change of variable $v(t)=u(t)-\zeta(t)$, inclusion (1) turns into

$$
\left\{\begin{array}{l}
\frac{d v}{d t} \in A v(t)+F(v(t)+\zeta(t))+\sum_{i=1}^{m} A \phi_{i} w_{i}(t), \\
v(0)=v_{0}=u_{0} .
\end{array}\right.
$$

Defining the multivalued map $\widetilde{F}:[0, T] \times \Omega \times X \rightarrow C_{v}(X)$, by $\widetilde{F}(t, \omega, x)=F(x+\zeta(t))+A \zeta(t)$, it is easy to obtain from (F2) that $\widetilde{F}$ satisfies (F1), (F2) and the next property: 
(F3) For any $x \in X$ there exists $n(\cdot) \in L_{1}(0, T)$ depending on $x$ and $\omega$ such that $\|\widetilde{F}(t, \omega, x)\|^{+} \leq$ $n(t)$, a.e. in $(0, T)$.

Consider also the problem

$$
\left\{\begin{array}{l}
\frac{d v(t)}{d t}=A v(t)+f(t), \\
v(0)=v_{0}
\end{array}\right.
$$

where $f(\cdot) \in L^{1}([0, T], X)$.

Definition 5. - The function $u:[0, T] \rightarrow X$ is called a strong solution of problem (3) if:

i) $u(\cdot)$ is continuous on $[0, T]$ and $u(0)=u_{0}$;

ii) $u(\cdot)$ is absolutely continuous on any compact subset of $(0, T)$ and almost everywhere (a.e.) differentiable on $(0, T)$;

iii) $u(\cdot)$ satisfies $(3)$ a.e. on $(0, T)$.

Definition 6. - The continuous function $v:[0, T] \rightarrow X$ is called an integral solution of problem (3) if $v(0)=v_{0}$ and

$$
\|v(t)-\xi\|^{2} \leq\|v(s)-\xi\|^{2}+2 \int_{s}^{t}\langle f(\tau)+A \xi, v(\tau)-\xi\rangle d \tau, t \geq s, \forall \xi \in D(A) .
$$

It is well known (see Barbu [4, p.124]) that any strong solution of problem (3) is an integral solution.

Definition 7. - The process $v:[0, T] \times \Omega \rightarrow X$ is said to be an integral solution of problem (2) if for any $\omega \in \Omega$ the map $v(\cdot)=v(\cdot, \omega):[0, T] \rightarrow X$ is continuous, $v(0)=v_{0}$, and for some selection $f \in L^{1}([0, T], X), f(t) \in \widetilde{F}(t, \omega, v(t))$ a.e. on $(0, T)$, the inequality (4) holds.

In what follows, we will omit $\omega$ if no confusion is possible.

If condition $(A)$ holds and $f \in L^{1}([0, T], X)$, then $\forall v_{0} \in \overline{D(A)}$ there exists a unique integral solution $v(\cdot)$ of (3) for each $T>0$ (see Barbu [4, p.124]). We shall denote this solution by $v(\cdot)=I\left(v_{0}\right) f(\cdot)$. If $(A),(F 1)-(F 3)$ hold, then $\forall v_{0} \in \overline{D(A)}$ there exists at least one integral solution $v(\cdot)=I\left(v_{0}\right) f(\cdot)$ of (2) for each $T>0$ (see Tolstonogov [15], Theorem 3.1), so each solution can be extended on $[0, \infty)$. Let us denote by $\mathcal{D}\left(v_{0}, \omega\right)$ the set of all integral solutions of (2) such that $v(0)=v_{0}$. We define the maps $G: \mathbf{R}^{+} \times \Omega \times \overline{D(A)} \rightarrow P(\overline{D(A)}), \theta_{s}: \Omega \rightarrow \Omega$ as follows

$$
\begin{gathered}
G(t, \omega) v_{0}=\left\{v(t)+\zeta(t) \mid v(\cdot) \in \mathcal{D}\left(v_{0}, \omega\right)\right\}, \\
\theta_{s} \omega=\left(w_{1}(s+\cdot)-w_{1}(s), \ldots, w_{m}(s+\cdot)-w_{m}(s)\right) \in \Omega .
\end{gathered}
$$

Then the function $\widetilde{\zeta}$ corresponding to $\theta_{s} \omega$ is $\widetilde{\zeta}(\tau)=\zeta(s+\tau)-\zeta(s)=\sum_{i=1}^{m} \phi_{i}\left(w_{i}(s+\tau)-w_{i}(s)\right)$.

Theorem 3. - Let $(A),(F 1),(F 2)$ hold. Then $G$ satisfies the cocycle property. Moreover, if the semigroup $S(t, \cdot)$ generated by the operator $A$ is compact, then $G$ generates a MRDS.

\subsection{Existence of a random attractor}

As in the deterministic case, a dissipative assumption will imply the existence of a compact absorbing set and, as a consequence of Theorem 2, the existence of the random attractor for the multivalued random semiflow. However, to this end more regularity of the integral solutions is needed.

Proposition 4. - Let $(A),(F 1),(F 2)$ hold. Suppose that each integral solution of (2), $v(\cdot)=$ $I\left(u_{0}\right) f(\cdot)$ is a strong solution of (3). Let there exist constants $\delta>0, M \geq 0$ such that $\forall u \in D(A)$, $y \in F(u)$,

$$
\langle y, u\rangle \leq(-\delta+\varepsilon)\|u\|^{2}+M
$$




\section{T. Caraballo, J.A. Langa, J. Valero}

where $\varepsilon \geq 0$ is the biggest constant such that

$$
\langle A u, u\rangle \leq-\varepsilon\|u\|^{2}, \forall u \in D(A)
$$

Then there exists a random radius $r(\omega)>0$ such that for $\mathbf{P}-$ almost all $\omega \in \Omega$ and any bounded set $B \subset \overline{D(A)}$ we can find $T(B)=T(B, \omega) \geq 1$ for which

$$
\left\|G\left(-1+t_{0}, \theta_{-t_{0}} \omega\right) u_{0}\right\|^{+} \leq r\left(\theta_{-1} \omega\right), \forall t_{0} \geq T(B), \forall u_{0} \in B
$$

THEOREM 5. - Let the conditions of Proposition 4 hold, the semigroup $S(t, \cdot)$ generated by the operator $A$ be compact and the multivalued map $G(1, \omega)$ be compact (that is, it maps bounded sets into precompact ones). Then, $G$ has the minimal global random attractor $\mathcal{A}(\omega)$. Moreover, it is measurable with respect to $\mathcal{F}$.

Remark. This abstract result is applied in [6] to stochastic reaction-diffusion inclusions.

Acknowledgement. The authors wish to thank Prof. J. Real for helpful suggestions on this work and for his invaluable help with the French version.

\section{References}

[1] Ahmed N.U., Nonlinear stochastic differential inclusions on Banach space, Stoch. Anal. Appl. 12 (1994), 1-10.

[2] Arnold L., Random Dynamical Systems, Springer Monographs in Mathematics (1998).

[3] Aubin J.P., Frankowska H., Set-Valued Analysis, Birkhäuser, Boston (1990).

[4] Barbu V., Nonlinear Semigroups and Differential Equations in Banach Spaces, Editura Academiei, Bucuresti (1976).

[5] Caraballo T., Langa J.A., Robinson J., Upper semicontinuity of attractors for small random perturbations of dynamical systems, Comm. Partial Differential Equations 23 (1998), 1557-1581.

[6] Caraballo T., Langa J.A., Valero J., Global attractors for multivalued random dynamical systems, to appear.

[7] Castaing C., Valadier M., Convex Analysis and Measurable Multifunctions, LNM Vol. 580, Springer (1977).

[8] Crauel H., Flandoli F., Attractors for random dynamical systems, Prob. Theory Related Fields 100 (1994), 365-393.

[9] Da Prato G., Frankowska H., A stochastic Filippov theorem, Stoch. Anal. Appl. 12 (1994), no. 4, $409-426$.

[10] Hale J., Asymptotic Behavior of Dissipative Systems, Math. Surveys and Monographs, AMS, Providence (1988).

[11] Ladyzhenskaya O., Attractors for Semigroups and Evolution Equations, Accademia Nazionale dei Lincei, Cambridge University Press, Cambridge (1991).

[12] Melnik V., Valero J., On Attractors of multivalued semi-flows and differential inclusions, Set-Valued Anal. 6 (1998), 83-111.

[13] Schmalfuss B., Backward cocycle and attractors of stochastic differential equations, in V. Reitmann, T. Redrich and N. JKosch (eds.), International Seminar on Applied Mathematics-Nonlinear Dynamics: Attractor Approximation and Global Behaviour (1992), 185-192.

[14] Temam R., Infinite Dimensional Dynamical Systems in Mechanics and Physics, Springer-Verlag, New York (1988).

[15] Tolstonogov A.A., On solutions of evolution inclusions.I, Sibirsk. Mat. Zh. 33, 3 (1992), 161-174 (English translation in Siberian Math. J., 33, 3 (1992)).

[16] Valero J., Finite and infinite-dimensional attractors of multivalued reaction-diffusion equations, Acta Math. Hungar. Vol. 88, no. 3 (2000), 239-258. 